\title{
Upper extremity surgery in younger children under ultrasound-guided supraclavicular brachial plexus block: a case series
}

\author{
Hamid Reza Amiri · Ramin Espandar
}

Received: 16 April 2010/ Accepted: 16 October 2010/Published online: 23 November 2010 (c) EPOS 2010

\begin{abstract}
Purpose Supraclavicular brachial plexus block is considered to be one of the most effective anesthetic procedures for upper extremity surgeries. Its major drawback is placement of the needle, with inaccurate placement, especially in children, being a risk factor for pneumothorax and vascular puncture and failure of the procedure. Ultrasoundguided needle placement may reduce the risk of complications and increase the accuracy of the block, particularly in pediatric patients. Little has been published on the efficacy and safety of ultrasound-guided supraclavicular block in children based on practical experience, and there has been no published report on its usage in younger children $(<6$ years old).

Methods Seventeen patients between the ages of 6 months and 6 years were randomly selected to test the efficacy of ultrasound-guided supraclavicular block in younger children. The ultrasound probe was used for proper placement of the needle. After confirmation of the needle location using a nerve locator, the anesthetic agent was injected. The procedure time, establishment time, duration of analgesia, any complications related to the procedure, and surgeon's satisfaction were recorded and assessed.
\end{abstract}

\section{H. R. Amiri}

Department of Anesthesiology, Imam University Hospital, Tehran University of Medical Sciences, Keshavarz Blvd., 1419733141 Tehran, Iran

\section{R. Espandar ( $\square)$}

Department of Orthopedic Surgery, Imam University Hospital, Tehran University of Medical Sciences, Keshavarz Blvd., 1419733141 Tehran, Iran

e-mail: espandar@gmail.com; espandarmd@sina.tums.ac.ir
Results The length of the procedure was $10.35 \pm 1.22 \mathrm{~min}$, establishment time was $89.59+18 \mathrm{~s}$, and the duration of the analgesia was between 6 and $16 \mathrm{~h}$ (mean $9.76 \pm 2.57 \mathrm{~h}$ ). The recovery time was $24.4+6.5$ min (range 15-37 min), and the duration of surgeries was $61.3 \pm 25.9$ min (range 15-110 $\mathrm{min}$ ). Not one procedure failed, and there was not one complication related to the procedure. The surgeon's satisfaction during surgery was good or excellent.

Conclusions The results of this study demonstrate the efficacy and safety of the ultrasound-guided supraclavicular brachial plexus block for orthopedic upper extremity surgeries in patients less than 6 years of age.

Keywords Pediatric upper extremity surgery .

Ultrasound - Supraclavicular - Brachial plexus block .

Pediatric anesthesia $\cdot$ Regional anesthesia

\section{Introduction}

Supraclavicular brachial plexus block is a known anesthetic option for upper limb surgery. While long-lasting pain relief and the possibility of earlier hospital discharge are two of the clinical benefits of this procedure, inconsistent block success remains one of its major limitations and can lead to an unplanned general anesthetic, increased material costs, and prolonged operating room time. Another limitation is the potential for procedure-related complications, such as nerve injury and unintentional vascular puncture. Children in particular frequently suffer from inadequate pain management during and especially after painful procedures, with an underestimation of the intensity of pain and fear of the risk of complications arising from the use of opioids possibly being important factors in this regard. Regional anesthesia may represent 
one of the best solutions for intraoperative and postoperative pediatric pain management; however, due to lack of proficiency and the increased risk of complications in children compared to adults, peripheral nerve blockade is not the method of choice for most of the anesthesiologists in children, especially among younger pediatric patients. In recent years, ultrasound guidance has been introduced to assist nerve localization and is increasingly being used for performing peripheral nerve blocks. Unlike traditional nerve localization techniques which rely on surface anatomical landmarks, patient's report of paresthesia, and/or elicitation of a motor response by electrical nerve stimulation, ultrasound guidance use sonoanatomic rather than a surface anatomy or neurophysiologic end point for block performance. However, nerve stimulation continues to be used along with ultrasound for additional confirmation of nerve identification [1]. Based on the surgeons' preference and the need for a tourniquet at the proximal part of the humerus in all surgeries despite the type of surgery, we consider supraclavicular block to be more suitable than more distal ones. This block can be performed with only one injection around the plexus, and it provides sufficient anesthesia at the tourniquet site as well.

Few studies have been published on upper extremity surgery under supraclavicular brachial plexus block in children. The aim of the study reported here was to demonstrate the efficacy and safety of ultrasound-guided supraclavicular brachial plexus blockade in children younger than 6 years of age.

\section{Materials and methods}

Following an explanation of the risks and benefits of ultrasound-guided supraclavicular brachial plexus block as the anesthetic procedure during surgery, the parents of 41 American Society of Anesthesiologists Physical Status (ASA-PS) I-II patients were asked to provide written informed consent for the use of this procedure as the method of anesthesia in their respective child's surgery. After permission had been received and exclusion criteria taken into account (consent/no consent; obvious or suspicious infection at the injection site), 17 patients were enrolled in the study. All interviews were carried out by one physician (HRA) at the Preoperative Anesthesia Clinic, and the parents had a minimum of $24 \mathrm{~h}$ to make their decision. The patients were between 6 months and 6 years of age, and all were scheduled for elective upper extremity surgery. The socio-demographic and clinical data for all patients are given in Table 1 .

After sedation with intranasal $(0.05-0.25 \mathrm{mg} / \mathrm{kg})$ Midazolam hydrochloride, the patient was transferred to the block room. A secure intravenous line was introduced, and supplemental oxygen (4-6 1/min) through a face mask given applied, followed by a bolus dose of $0.5 \mathrm{mg} / \mathrm{kg}$ of

Table 1 Socio-demographic and clinical information on the patients

\begin{tabular}{|c|c|c|c|c|c|c|c|c|}
\hline Procedure & $\operatorname{Sex}^{\mathrm{a}}$ & $\begin{array}{l}\text { Age } \\
\text { (months) }\end{array}$ & ASA-PS & $\begin{array}{l}\text { Procedure } \\
\text { time (min) }\end{array}$ & $\begin{array}{l}\text { Establishment } \\
\text { time(s) }\end{array}$ & $\begin{array}{l}\text { Duration of } \\
\text { analgesia }(\mathrm{h})\end{array}$ & $\begin{array}{l}\text { Recovery } \\
\text { time }\end{array}$ & $\begin{array}{l}\text { Duration of } \\
\text { surgery (min) }\end{array}$ \\
\hline Syndactyly repair & $\mathrm{F}$ & 8 & 2 & 10 & 83 & 16 & 19 & 47 \\
\hline Cleft hand repair & $\mathrm{F}$ & 6 & 1 & 14 & 94 & 11 & 32 & 95 \\
\hline Policization & $\mathrm{F}$ & 6 & 1 & 11 & 67 & 8.5 & 16 & 102 \\
\hline Forearm exostosis excision & $\mathrm{F}$ & 14 & 2 & 10 & 140 & 10 & 22 & 50 \\
\hline Trigger finger repair & $\mathrm{F}$ & 23 & 1 & 10 & 93 & 6.5 & 17 & 27 \\
\hline Forearm exostosis excision & M & 48 & 1 & 11 & 81 & 12 & 19 & 53 \\
\hline Pollicization & M & 11 & 2 & 10 & 87 & 10 & 15 & 110 \\
\hline Syndactyly repair & M & 18 & 1 & 12 & 72 & 8.5 & 27 & 65 \\
\hline CRIF of distal radius & $\mathrm{F}$ & 8 & 2 & 10 & 78 & 11 & 23 & 50 \\
\hline Cleft hand repair & $\mathrm{F}$ & 6 & 2 & 9 & 100 & 12 & 33 & 105 \\
\hline Syndactyly repair & $\mathrm{F}$ & 12 & 1 & 10 & 75 & 6 & 28 & 52 \\
\hline Syndactyly repair & $\mathrm{F}$ & 19 & 1 & 9 & 80 & 12 & 37 & 61 \\
\hline CRIF of distal radius & M & 51 & 1 & 10 & 90 & 6 & 21 & 47 \\
\hline Syndactyly repair & $\mathrm{F}$ & 18 & 1 & 10 & 98 & 6 & 31 & 46 \\
\hline Syndactyly repair & $\mathrm{F}$ & 18 & 2 & 11 & 70 & 8.5 & 29 & 55 \\
\hline CRIF of distal radius & $\mathrm{F}$ & 69 & 2 & 9 & 110 & 9 & 24 & 53 \\
\hline Fracture of proximal phalanx & M & 70 & 1 & 10 & 105 & 11 & 22 & 25 \\
\hline
\end{tabular}

ASA-PS American Society of Anesthesiologists-physical status; CRIF closed reduction and internal fixation

${ }^{\text {a }} F$ female, $M$ male 
propofol (Propofol 1\% MCT/LCT Fresenius; Fresenius Kabi Austria, Graz, Austria) injected intravenously. Preparation and draping of the neck and supraclavicular region of the patient was done with povidone iondine solution $10 \%$. The ultrasound-guided supraclavicular block was modified from the technique originally described by Chan [2]. The sonographic view of the brachial plexus was obtained with a 9-MHz, 40-mm linear transducer (PB-L59EC-N; SonoAce PICO, Medison, South Korea) of a digital portable ultrasound unit equipped with spectral, color, and wave Doppler (SonoAce PICO, Medison, South Korea). The transducer was oriented transversally in the supraclavicular fossa just above the clavicle. In this view, the brachial plexus is superficial and lateral to the subclavian artery and visualized as a group of hypoechoic beans (Fig. 1). The location of the subclavian artery is confirmed by a pulse-wave Doppler image (Fig. 2). The

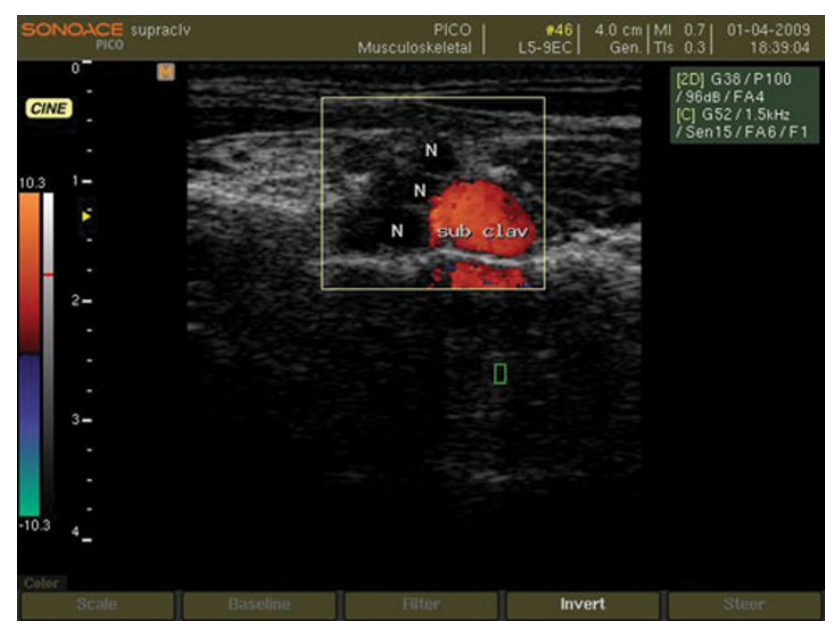

Fig. 1 Ultrasound view of brachial plexus in supraclavicular region. $N$ Brachial plexus, sub clav subclavian artery

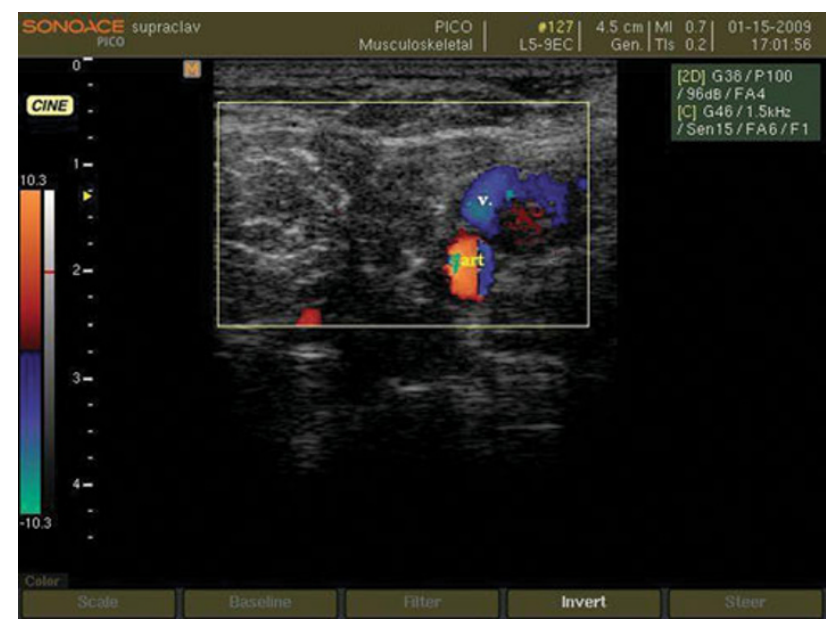

Fig. 2 The location of the subclavian artery (art) and vein $(v)$ in supraclavicular region are confirmed by Doppler ultrasonography supraclavicular block was performed using an insulated 35-mm, 24-gauge needle with a $20^{\circ}$ cutting bevel (Polymedic UPC, TeMe Na, Carrieres sur Seine, France) under ultrasonographic guidance. The insulated needle was attached to the nerve locator (Polystim II; Polymedic UPC) and then introduced through the skin lateral to the probe and in a parallel manner to the ultrasonic beams in order to have a full-length view of the needle and its pathway throughout the procedure (Fig. 3). We considered a motor response to $0.5 \mathrm{~mA}$ of electrical impulse generated by an electrical nerve locator as confirmation that the tip of the needle was sufficiently close to the plexus [3-5]. Thereafter, the nerve locator was adjusted to $1 \mathrm{~Hz}, 0.5 \mathrm{~mA}$, and $0.1 \mathrm{~ms}$ during the procedure. The needle was advanced toward the brachial plexus, and when the tip was visualized adjacent to the hypoechoic beans, it was gently moved to produce a regular muscle twitch. A mixture of lidocaine hydrochloride $0.7 \%$ and bupivacaine hydrochloride $0.17 \%$ (Bupivacaine $0.5 \%$; Merck, Lyon, France) was injected at the site after frequent negative aspirations. During the injection procedure, the dispersion of the drug around the plexus was visualized through the sonographic view as a hypoechoic area; if indicated, the tip of the needle was transposed to produce a suitable distribution of local anesthetic around the plexus. The injection was stopped when the plexus was surrounded completely by the hypoechoic area (indicating local anesthetic distribution) or a total of $5 \mathrm{mg} / \mathrm{kg}$ lidocaine had been administered [6]. Throughout the procedure, blood pressure (non-invasive), surface body temperature, oxygen saturation (pulse oximetry), and end tidal $\mathrm{CO}_{2}$ (side stream capnometry) were monitored.

"Procedure time" was defined as the time between skin preparation and needle extraction and was measured in minutes by a nurse anesthetist. Time between the completion of the injection and unresponsiveness to painful stimuli

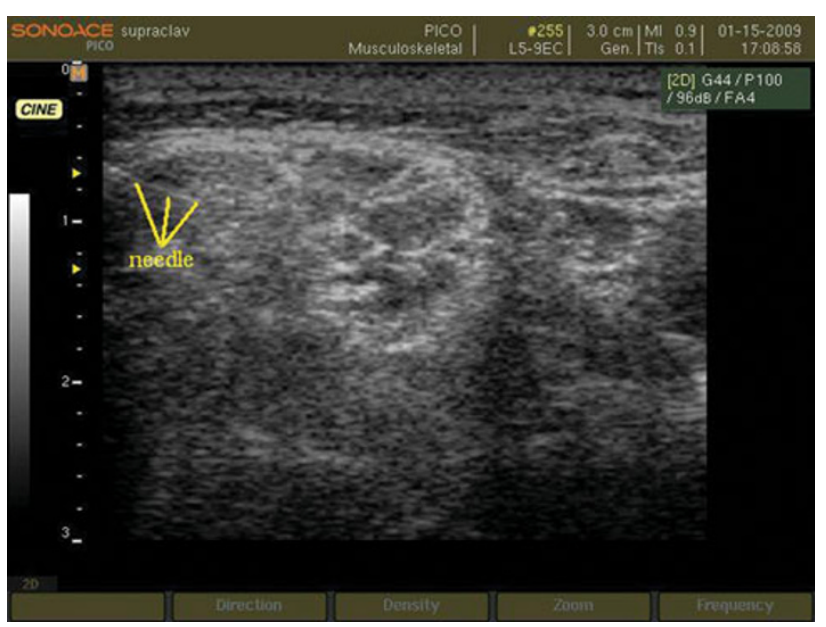

Fig. 3 Advancement of the needle toward the brachial plexus 
on the dependent limb (at the surgical site) was considered to be the "establishment time" and was recorded in seconds by an anesthesia resident who was blind to the study. The results are reported as the mean \pm standard deviation (SD). Responsiveness to painful stimuli up to $10 \mathrm{~min}$ after local anesthetic injection was considered as a block failure and assessed by the same anesthesia resident. In the case of failure, a second method of anesthesia was planned; otherwise, during the surgical procedure, clinical sedation was provided primarily with a propofol infusion, initially at $30-50 \mu \mathrm{g} / \mathrm{kg} / \mathrm{h}$, gradually decreased to $20-30 \mu \mathrm{g} / \mathrm{kg} / \mathrm{h}$ after $30 \mathrm{~min}$ and continued to the end of surgery. During surgery, pain was assessed based on hemodynamic changes (tachycardia, increased blood pressure) after other common causes, such as hypoxia, hypercapnia, hypothermia, and hypovolemia, were ruled out. A positive response to analgesic administration (decreased heart rate blood pressure) was regarded as block failure, and the anesthesia plan was altered once again. Following the surgical procedure, the infusion of sedative was stopped and the patient transferred to the recovery room. Our criteria for discharge from the recovery room was according to protocols in place and include an evaluation of level of consciousness and awakeness (in children: eye opening, crying, and body movements), vital signs (including pain) and $\mathrm{SpO}_{2}$ without supplemental $\mathrm{O}_{2}$. The patients were monitored carefully in the recovery room and also evaluated for pain; they were delivered to the ward under the supervision of a skilled nurse in the company of their parents. The recovery time was $24.4 \pm 6.5 \mathrm{~min}$ (range 15-37 min) (Table 1) About $2 \mathrm{~h}$ after discharge from the recovery room, oral nutrition was resumed (breast feeding in younger and fluids in the older ones). It is a common observation that most children fall asleep at this stage, necessitating that they be woken for an evaluation of motor function. This was not acceptable by our patients' parents, so an evaluation of the duration of the motor block (muscle relaxation) was not performed.

"Duration of analgesia" was considered as the time to appear pain considering behavioral scale based on parental report [7-12], the first dose of rescue analgesia, hemodynamic changes due to pain or any complication such as limb irritability or sensitivity due to dysesthesia or partial or complete immobility (paresis/paralysis) were recorded in hours by a blinded orthopedic resident.

\section{Results}

Seventeen pediatric patients [ 5 boys (29.4\%) and 12 girls $(70.6 \%)$ ], with a mean age of $23.8 \pm 21.6$ months (range 6 months to 6 years), were enrolled in the study. Ten patients (58.82\%) were in ASA-PS I and seven patients $(41.18 \%)$ were in ASA-PS II. The duration of analgesia ranged from 6 to $16 \mathrm{~h}$ (mean $9.76 \pm 2.57 \mathrm{~h}$ ). No blocks failed either before or during the surgical procedures. The surgeon's satisfaction regarding the anesthesia during operation was recorded as excellent in 11 and good in six cases. The procedure time was between 9 and $14 \mathrm{~min}$ (mean $10.35 \pm 1.22 \mathrm{~min}$ ) while establishment time was between 67 and $140 \mathrm{~s}$ (mean $89.59+17.97 \mathrm{~s}$ ). The mean duration of surgeries was $61.35 \pm 26 \mathrm{~min}(25-110 \mathrm{~min})$ (Table 1). No complications related to the anesthetic technique arose during the study. Patients' data, including surgical procedures, are summarized in Table 1.

\section{Discussion}

Pneumothorax and vascular puncture are the most potentially life-threatening complications that can arise with the use of the supraclavicular brachial plexus block [13, 14]. The consequent risk of pneumothorax is higher in pediatric groups because of the anatomic proximity of the cervical pleura [15]. Another main complication of blind approaches results from the spread of high volumes of local anesthetic [16]. For these reasons, traditional methods of supraclavicular block of the brachial plexus have not been recommended for use in pediatric patients [17]. The development of the real-time ultrasound guidance procedure has dramatically reduced the risks of pneumothorax and vascular puncture as the surrounding structures (pleura, subclavian artery and vein) are visualized [6]. As a result, the spread of local anesthetic around the target nerve can be assessed and more precisely administered at the correct location [18]. In addition, block success rate has increased from approximately $85 \%$ using the landmark technique to $95 \%$ using ultrasound guidance $[19,20]$. Ultrasound guidance for regional nerve blocks is rapidly becoming the standard of care in regional anesthesia [21], and ultrasound guidance has been introduced into clinical practice as a possible option to identify peripheral nerves, providing the potential advantage of optimizing the dispersion of the local anesthetic solution around the nerves under sonographic visualization [22]. Since the first study describing the use of ultrasound guidance in pediatric regional anaesthesia reported by Marhofer et al. [23], who observed an onset time of 9 min for infraclavicular blocks in children with ropivacaine $0.5 \%$ in a randomized clinical trial, there have only been a limited number of randomized control trails in children comparing ultrasound guidance peripheral nerve block with other techniques [24]. Available evidence in children demonstrates that ultrasoundguided peripheral nerve blocks improve the quality, onset, duration, and success rate of nerve blocks and help to lower the local anesthetic volume needed to perform the blocks $[23,25,26]$. To the best of our knowledge, our study is the 
first published English-language report on the use of ultrasound-guided supraclavicular brachial plexus block in children younger than 6 years of age. Our results are comparable with those of other studies that have used the same technique for supraclavicular block in adults and older children. In comparison to other studies related to pediatric regional anesthesia, we used lower concentrations of local anesthetics to obtain acceptable results, which seem to be important in terms of the risk of systemic toxicity, especially in the pediatric age group [27]. In addition, postoperative analgesia was considerable and satisfactory.

Based on our results, we conclude that ultrasoundguided supraclavicular brachial plexus nerve block is a safe, convenient, and effective anesthesia option for upper extremity surgical procedures in younger children, with reasonable procedure, establishment, and analgesia times. Further comparative studies with a much larger group of patients are required for a careful evaluation of the risks and benefits of this procedure, especially in the younger pediatric patient group.

\section{References}

1. Gray AT (2006) Ultrasound-guided regional anesthesia: current state of the art. Anesthesiology 104(2):368-373

2. Chan VW, Perlas A, Rawson R et al (2003) Ultrasound-guided supraclavicular brachial plexus block. Anesth Analg 97(5): 1514-1517

3. Bashein G, Haschke RH, Ready LB (1984) Electrical nerve location: numerical and electrophoretic comparison of insulated vs. un-insulated needles. Anesth Analg 63(10):919-924

4. Keschner MT, Michelsen H, Rosenberg AD et al (2006) Safety and efficacy of the infraclavicular nerve block performed at low current. Pain Pract 6(2):107-111

5. Sinha SK, Abrams JH, Weller RS (2007) Ultrasound-guided interscalene needle placement produces successful anesthesia regardless of motor stimulation above or below $0.5 \mathrm{~mA}$. Anesth Analg 105(3):848-852

6. Chan VW, Perlas A, McCartney CJ et al (2007) Ultrasound guidance improves success rate of axillary brachial plexus block. Can J Anaesth 54(3):176-182

7. Li L, Liu X, Herr K (2007) Postoperative pain intensity assessment: a comparison of four scales in Chinese adults. Pain Med 8(3):223-234

8. Beyer JE, McGrath PJ, Berde CB (1990) Discordance between self-report and behavioral pain measures in children aged 3-7 years after surgery. J Pain Symptom Manage 5(6):350-356
9. Jacobson SJ, Kopecky EA, Joshi P et al (1997) Randomised trial of oral morphine for painful episodes of sickle-cell disease in children. Lancet 350(9088):1358-1361

10. McGrath PJ, Rosmus C, Camfield C et al (1998) Behaviours caregivers use to determine pain in non-verbal, cognitively impaired individuals. Dev Med Child Neurol 40:340-343

11. McGrath PJ, McAlpine L (1993) Physiologic perspectives on pediatric pain. J Pediatr 122(5 Pt 2):S2-S8

12. Mitchell P (1999) Understanding a young child's pain. Lancet 354(9191): 1708

13. Pande R, Pande M, Bhadani U et al (2000) Supraclavicular brachial plexus block as a sole anaesthetic technique in children: an analysis of 200 cases. Anaesthesia 55(8):798-802

14. Tobias JD (2001) Brachial plexus anaesthesia in children. Paediatr Anaesth 11(3):265-275

15. Suresh S, Wheeler M (2002) Practical pediatric regional anesthesia. Anesthesiol Clin North America 20(1):83-113

16. De José María B, Banús E, Navarro Egea M et al (2008) Ultrasound-guided supraclavicular vs infraclavicular brachial plexus blocks in children. Paediatr Anaesth 18(9):838-844

17. Dalens B (1995) Proximal blocks of the upper extremity: benefit/ risk ratio. In: Dalens B (ed) Regional anaesthesia in infants, children and adolescent. Williams and Wilkins, Baltimore, pp 275-314

18. Riazi S, Carmichael N, Awad I et al (2008) Effect of local anesthetic volume $(20 \mathrm{vs} .5 \mathrm{ml})$ on the efficacy and respiratory consequences of ultrasound-guided interscalene brachial plexus block. Br J Anaesth 101(4):549-556

19. Kapral S, Krafft P, Eibenberger K et al (1994) Ultrasound-guided supraclavicular approach for regional anesthesia of the brachial plexus. Anesth Analg 78(3):507-513

20. Williams SR, Chouinard P, Arcand G et al (2003) Ultrasound guidance speeds execution and improves the quality of supraclavicular block. Anesth Analg 97(5):1518-1523

21. Stone MB, Wang R, Price DD (2008) Ultrasound-guided supraclavicular brachial plexus nerve block vs. procedural sedation for the treatment of upper extremity emergencies. Am J Emerg Med 26(6):706-710

22. Casati A, Danelli G, Baciarello M et al (2007) A prospective, randomized comparison between ultrasound and nerve stimulation guidance for multiple injection axillary brachial plexus block. Anesthesiology 106(5):992-996

23. Marhofer P, Sitzwohl C, Greher M et al (2004) Ultrasound guidance for infraclavicular brachial plexus anaesthesia in children. Anaesthesia 59(7):642-646

24. Rubin K, Sullivan D, Sadhasivam S (2009) Are peripheral and neuraxial blocks with ultrasound guidance more effective and safe in children? Paediatr Anaesth 19(2):92-96

25. Oberndorfer U, Marhofer P, Bösenberg A et al (2007) Ultrasonographic guidance for sciatic and femoral nerve blocks in children. Br J Anaesth 98(6):797-801

26. Willschke H, Marhofer P, Bösenberg A et al (2005) Ultrasonography for ilioinguinal/iliohypogastric nerve blocks in children. Br J Anaesth 95(2):226-230

27. Marhofer P, Chan VW (2007) Ultrasound-guide regional anesthesia: current concepts and future trends. Anesth Analg 104(5): $1265-1269$ 